\title{
Editorial
}

\section{Compact Microstrip Antenna Structures with Multiband, Broadband, and Band-Notched Properties, for Portable Devices}

\author{
Alistair P. Duffy, ${ }^{1}$ Mohammad Naser-Moghadasi, ${ }^{2}$ \\ Jalil Rashed-Mohassel, ${ }^{3}$ and Bal Singh Virdee ${ }^{4}$ \\ ${ }^{1}$ De Montfort University, Leicester LE1 9BH, UK \\ ${ }^{2}$ Islamic Azad University, Science and Research Branch, Tehran, Iran \\ ${ }^{3}$ University of Tehran, Iran \\ ${ }^{4}$ London Metropolitan University, UK \\ Correspondence should be addressed to Alistair P. Duffy; apd@dmu.ac.uk
}

Received 18 March 2014; Accepted 18 March 2014; Published 14 April 2014

Copyright (C) 2014 Alistair P. Duffy et al. This is an open access article distributed under the Creative Commons Attribution License, which permits unrestricted use, distribution, and reproduction in any medium, provided the original work is properly cited.

It is difficult to list all the current applications for microstrip antennas; it could be said that they are the transducers that control modern life. Yet, every year, new applications are required which challenge the performance limits of current designs. Some of those challenges are associated with controlling the spectrum performance and continuing to seek techniques to tailor the shape to the applications.

This special issue brings some of the latest thinking in compact microstrip antenna design. However, the variety of approaches presented in the papers shows the possibilities that exist for future developments: this special issue is as much about setting out possibilities as it is about presenting new thinking.

The papers in this special issue are as follows. An approach to design microstrip antennas is presented in "Design of arbitrarily shaped planar microstrip antenna arrays with improved efficiency" which considers improved radiation efficiency. Focusing on the application areas of Bluetooth and WLAN, a dual-band planar inverted-E antenna (PIEA) is presented in "Compact dual-band planar inverted-eshaped antenna using defected ground structure" with a novel ground. A mesh grid structure planar wideband zero index metamaterial (ZIM) antenna is presented in "Zero index metamaterial for designing high-gain patch antenna" that provides a bandwidth of $9.9 \mathrm{GHz}$ to $11.4 \mathrm{GHz}$.An arc $\mathrm{H}$-shaped slot on the radiating patch provides dual bandnotched characteristics which is discussed in "Design and analysis of a novel dual band-notched UWB antenna."
The paper entitled "Printed modified bow-tie dipole antenna for DVB/WLAN applications" presents a printed modified bow-tie dipole antenna which consists of asymmetric feed and inserted slots. A frequency range of 3.1$10.6 \mathrm{GHz}$ is achieved by an antenna of only $30 \mathrm{~mm} \times$ $36 \mathrm{~mm}$ and is discussed in "A compact UWB diversity antenna." A modified compact planar ultrawideband (UWB) monopole antenna with triple controllable band-notched characteristics is presented in "A compact planar UWB antenna with triple controllable band-notched characteristics," which consists of a modified staircased V-shaped radiating element and partial ground plane. The radiation pattern is controlled using elliptical and rectangular patches, which is discussed in "A compact size 4-19.1 GHz heart shape UWB antenna with triangular patches."

\section{Acknowledgments}

The editors would like to extend their gratitude to the many anonymous referees and to the authors for responding so positively to the questions asked and the suggestions made by those referees.

Alistair P. Duffy

Mohammad Naser-Moghadasi Jalil Rashed-Mohassel

Bal Singh Virdee 

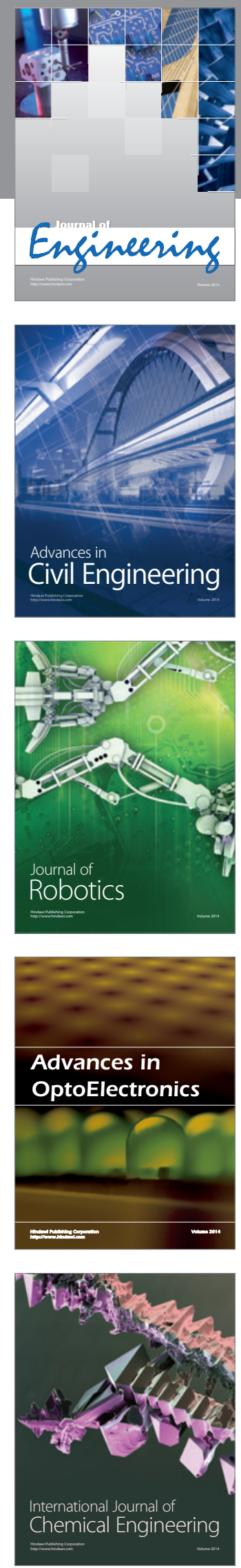

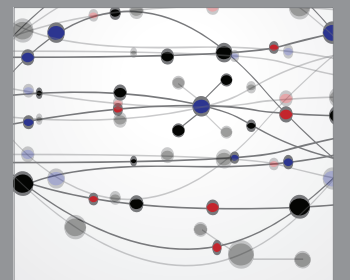

The Scientific World Journal
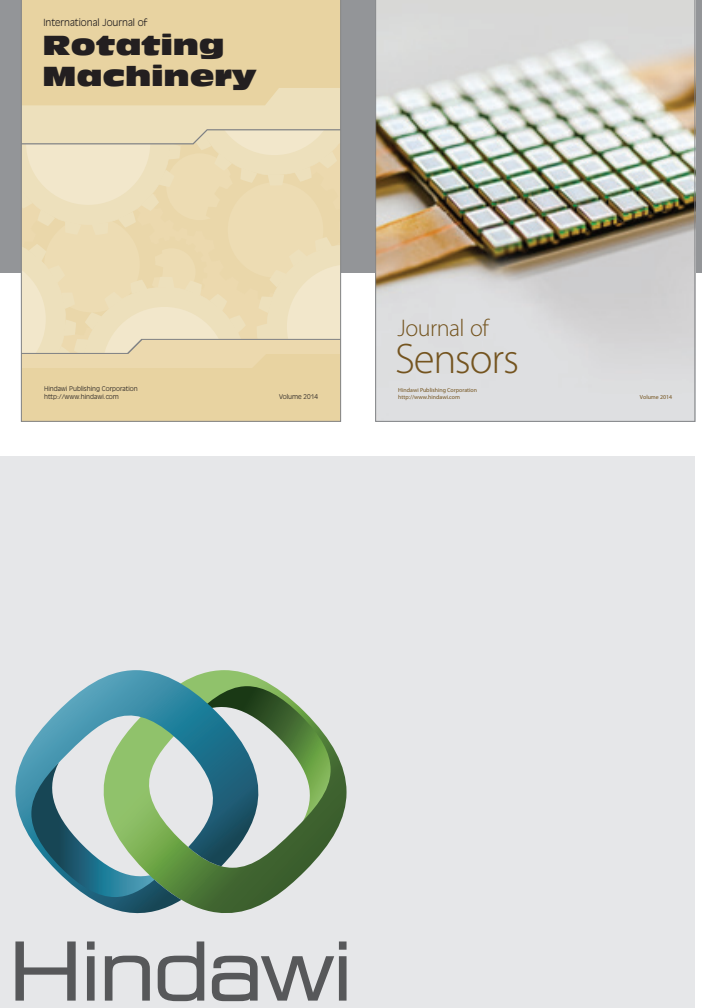

Submit your manuscripts at http://www.hindawi.com
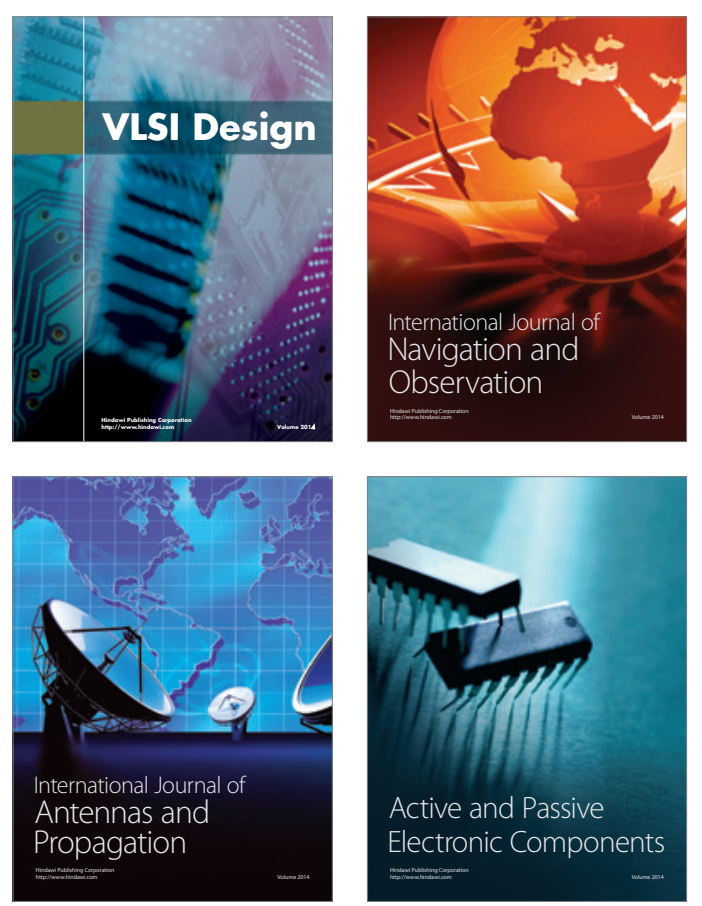
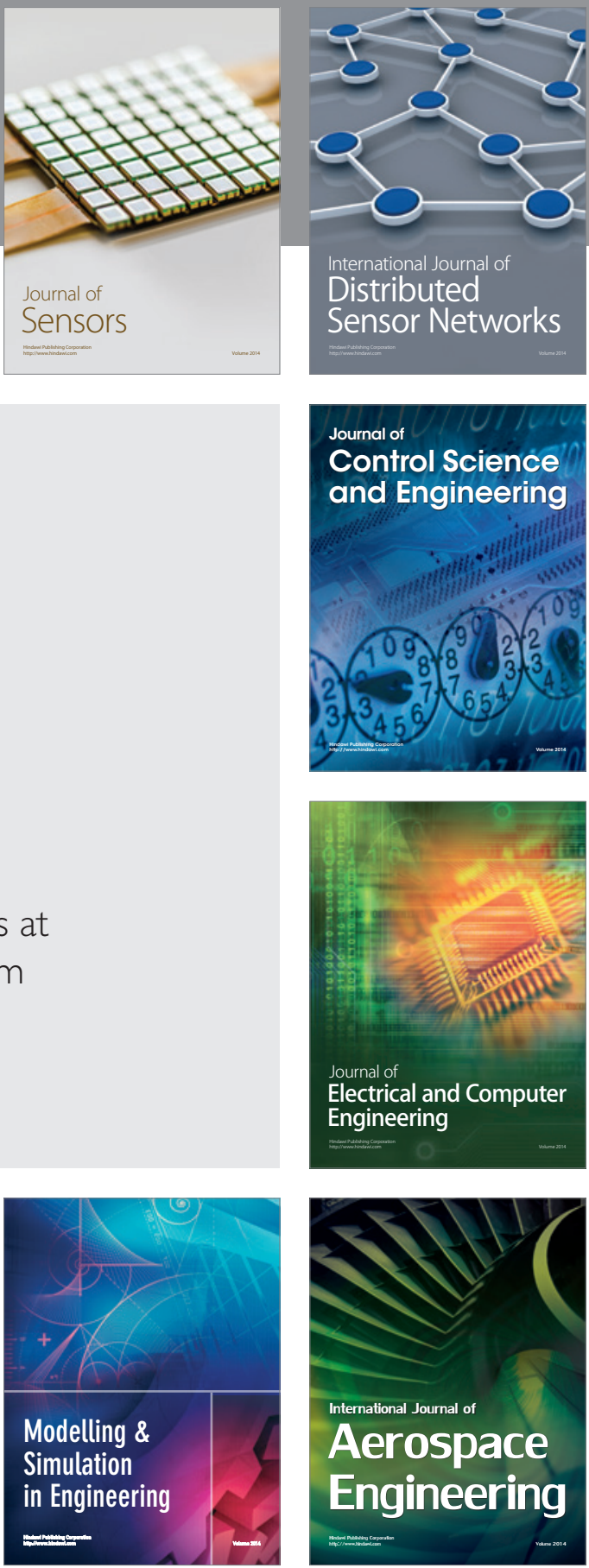

Journal of

Control Science

and Engineering
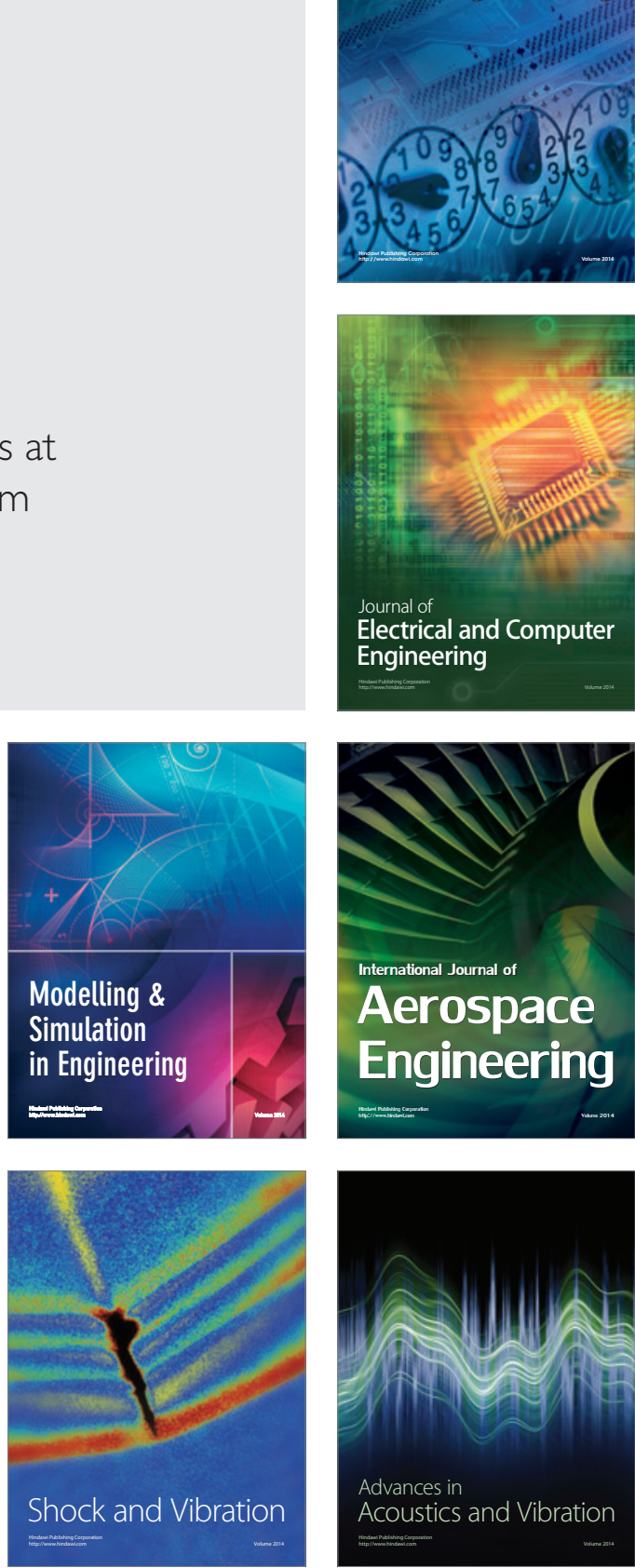